\section{Asthma-Selbst-Management mit dem Ampelschema: Welcher Peakflow, welche Guidelines?}

Dieser Text versteht sich als ein Diskussionsbeitrag zur Debatte über den Nutzen und die Praktikabilität eines regelmäßigen Peak flow-Monitorings bei Patienten mit Asthma bronchiale.

Beim Asthma handelt es sich um eine chronisch entzündliche Erkrankung. Exazerbationen treten sehr viel häufiger auf, als z. B. bei der Polyarthritis. Im Gegensatz zum Rheuma sollte beim Asthma nicht nur der Arzt Exazerbationen erkennen und darauf reagieren können, sondern auch der Patient. Eine optimale Therapie, die dem Asthmatiker größtmögliche Freiheit in bezug auf Beruf, Hobby und Ferien gewährt und die Wahrscheinlichkeit von Hospitalisationen reduziert, ist nur durch die Miteinbindung der Patienten möglich.

Eine Wissensvermittlung im Rahmen einer Patientenschulung ist Voraussetzung dafür, daß Patienten Mitverantwortung übernehmen können. Die Schulung sollte aus Gründen der Qualitätssicherung auf festgelegten Standards basieren, die in Form internationaler Guidelines vorliegen. Ein wesentlicher Bestandteil der Schulung von Asthma-Patienten ist das Erlernen von Peak flow-Messungen. Die Zweckmäßigkeit eines regelmäßigen Peak flow-Monitorings wird in der Literatur zwar kontrovers diskutiert [1,2], für Patienten mit mittelschwerem bis schwerem Asthma jedoch überwiegend befürwortet $[3,4]$ und entsprechend in den wichtigsten Guidelines empfohlen. Objektive Meßwerte zur Erfassung einer Exazerbation sind für Patienten deshalb wichtig, da besonders diejenigen mit schwerem Asthma den Grad der bronchialen Obstruktion häufig unterschätzen [5] und durch den Einsatz langwirkender Betastimulatoren typische Symptome einer beginnenden Exazerbation lange Zeit unterdrückt werden können [6].

Zur vereinfachten Interpretation der Peak flow-Werte wurde das „Ampelschema“ entwickelt. Die Ampelfarben Grün, Gelb und Rot definieren bestimmte Bereiche von Peak flowWerten. Liegen die Meßwerte im grünen Bereich, bedeutet dies „weiterfahren“ mit der Basismedikation. Gelb steht für „Vorsicht“ und kurzfristige Therapieintensivierung, Rot für „Halt“ und Befolgen eines Notfallplanes.

Das Asthma-Selbst-Management auf der Basis täglicher Peak flow-Kontrollen und des Ampelschemas wurde bisher nur wenig validiert. In den durchgeführten Studien hat es sich als

Pneumologie 53 (1999) 626-629

(c) Georg Thieme Verlag Stuttgart · New York

\section{T. Rothe}

Luzerner Höhenklinik, Montana fehleranfällig erwiesen [7,8]. Die Ampelschemata der verschiedenen internationalen Guidelines differieren erheblich [9], insbesondere im Hinblick auf den Zeitpunkt der Peak flow-Messungen, die Grenzen der Ampelfarben und die empfohlenen therapeutischen Interventionen. Es ist nicht geklärt, welche Sensitivität und Spezifität die jeweiligen Obergrenzen der gelben bzw. roten Phase besitzen, um eine asthmatische Exazerbation zuverlässig zu erkennen. Die Unterschiede in den Guidelines sind darauf zurückzuführen, daß die verschiedenen Empfehlungen Meinungen wiedergeben. Überzeugende Studien, die einen klaren Vorteil der einen gegenüber der anderen Version aufzeigen würden, fehlen noch.

Die ersten Publikationen, in denen das Ampelschema zur Interpretation von Peak flow-Werten angewendet worden war, erschienen Ende der 80er Jahre. Die SouthamptonGuidelines [10] definierten die Obergrenze der gelben Phase bei $70 \%$, die der roten bei $50 \%$ des individuellen besten Peak flow-Wertes. In der gelben Phase, d.h. beim Unterschreiten der 70\%-Perzentile, wurde eine kurzfristige Verdopplung der Dosis topischer Kortikosteroide empfohlen. Zur Interpretation wurden nur die Peak flow-Werte herangezogen, die am frühen Morgen, d.h. vor Betastimulation, gemessen wurden. Die im gleichen Jahr erarbeiteten kanadischen Guidelines [11] gingen von anderen Grenzen der Ampelphasen (Gelb bei 85\%, Rot bei $60 \%$ ) aus und beruhten explizit auf den besten Peak flow-Werten, die im Laufe eines Tages gewonnen werden können, d.h. auch solchen nach Betastimulation.

Auf welchen Überlegungen basieren diese divergierenden Ansätze? Morgenmessungen sind sehr sensitiv zur Erfassung einer auch nur leichtgradigen Verschlechterung des Asthmas [12]. Eine Zunahme der bronchialen Hyperreaktivität führt konsekutiv zu vermehrter Peak flow-Variabilität mit tiefen Peak flow-Werten am frühen Morgen (morning dipping). Therapeutisch sind in dieser Situation topische Kortikosteroide systemischen vorzuziehen, da damit die bronchiale Hyperreaktivität besser beeinflußt werden kann [13]. Nachteilig ist jedoch, daß im Falle einer Medikation mit langwirkenden Betastimulatoren eine langsame Verschlechterung des Asthmas maskiert werden kann, da die Peak flow-Variabilität dadurch lange gering bleibt [6]. Überdies wird vernachlässigt, inwieweit eine nachgewiesene bronchiale Obstruktion auf einem Bronchospasmus, oder auf einer entzündlichen Schwellung der Mukosa beruht. Es hat aber erhebliche therapeutische Konsequenzen, ob ein Morgen Peak flow von $60 \%$ des individuellen Bestwerts spontan im Tagesverlauf bzw. auf Betastimulation Reversibilität zeigt oder nicht. Hält 
sich ein Asthmatiker an ein Ampelschema auf der Basis von Peak flow-Werten am frühen Morgen, kommt es oft zu einem „overtreatment“ im Hinblick auf den Einsatz oraler Steroide [8].

Ampelschemata, die auf Werten nach Betastimulation beruhen, erfassen, ob nach Betastimulation noch eine residuelle Obstruktion im Sinne einer entzündlichen Schleimhautschwellung verbleibt. Sie kranken aber daran, daß die $\mathrm{Zu}$ nahme der Peak flow-Variabilität als frühes Zeichen einer Verschlechterung verkannt wird.
Die ersten internationalen Guidelines, die das Ampelschema aufnahmen, war der ICR 1992 [14]. Die Peak flow-Grenzen lagen dort bei $80 \%$ für Gelb bzw. $50 \%$ für Rot. Dort wurde schon für die gelbe Phase eine Intervention mit oralen Kortikosteroiden vorgeschlagen. Die Autoren empfahlen, entweder die Peak flow-Werte vor, bzw. nur die nach Betastimulation zur Interpretation im Rahmen des Ampelschemas heranzuziehen. Dies macht insofern wenig Sinn, da die empfohlenen Grenzen der Ampelphasen für beide Vorgehensweisen dieselben waren. Eine Validierung fiel entsprechend enttäuschend aus [7].

\section{Ampelschema zur Selbst-Erkennung einer Verschlimmerung des Asthmas}

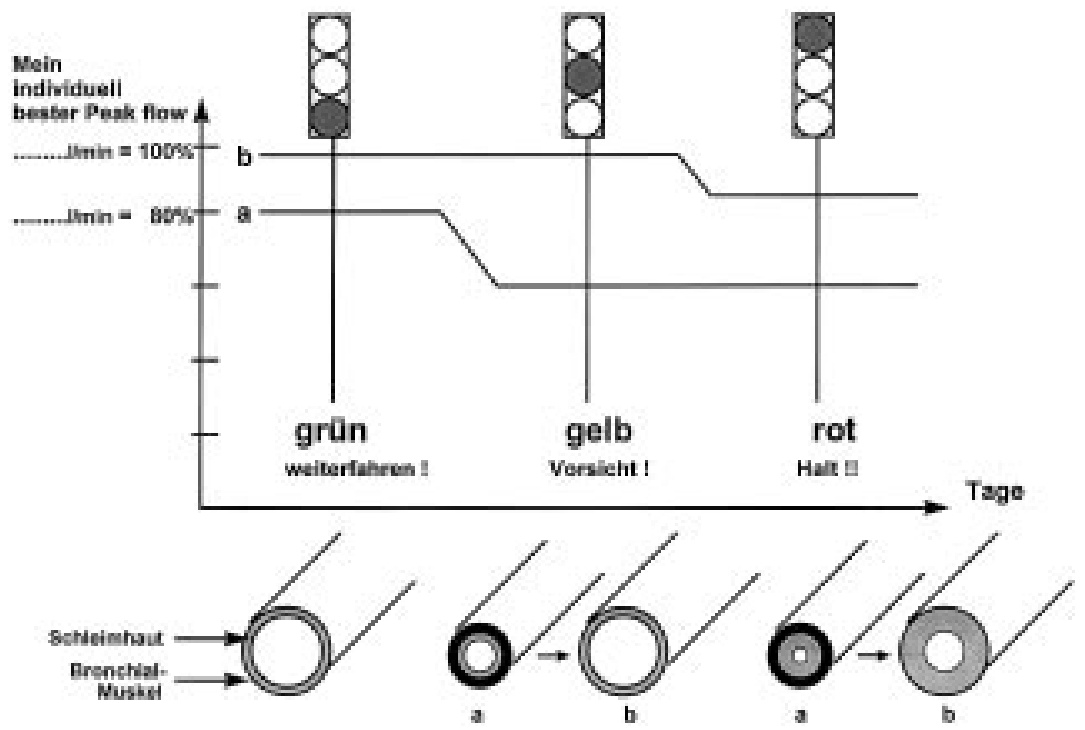

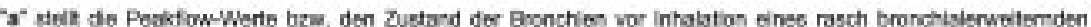

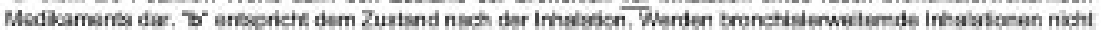

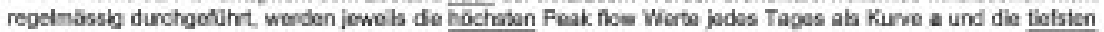
Werte pdes Tapes als Kirve b mbeinsnder verbunden. Solhe der Peak flow ekmal unier eos abtalen, oder im

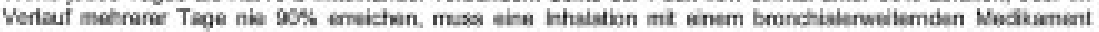
arfoloen. Ein Woches in einen anderen Farbbereich ist dann eingetroten, menn die Poak flow.Worto mindestena für 2 Tage unter die gensenten Grenven sbgetallen elnd.
Medikamente Grün
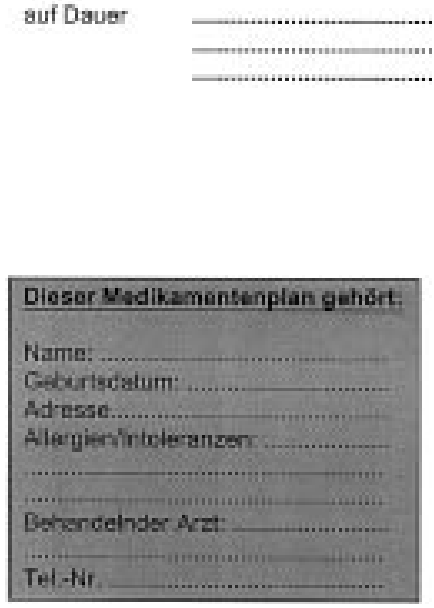

auf Dauer
Gelb

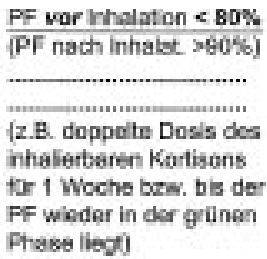

\section{Rot}

PF nach inhal. $<90 \%$

[2.8. Vertoppeing der Dasis des inhaliertaren Kortisoreraparates?]

PF nach inhal < toos

iz.B. Prednison - Tabl. $2 \times 20 \mathrm{mg}$ lol. fur $5 \mathrm{Tg}$. bzw. bis PF wieder in der geben Phase)
NotfallPF nach Inhal $<50 \%$

(Sofart Arztbesuch II)
Abb. 1 Graphische Interpretationshilfe von Peak flow-Werten auf der Basis des Ampelschemas. (modifiziert nach T. Rothe: „Modernes Asthma-Management“. Huber Verlag Bern 1998. Mit freundlicher Genehmigung des Verlages) 
1995 erschienen die GINA-Richtlinien [15], die zwar im November 1998 überarbeitet wurden, sich im Hinblick auf die Empfehlungen zum Ampelschema jedoch nicht von der Version 1995 unterscheiden. In diesen Richtlinien heißt es, Peak flow-Messungen sollten idealerweise zweimal täglich, am frühen Morgen und 10 bis 12 Stunden später, durchgeführt werden. Und zwar vor und nach Betastimulation, sofern ein Betastimulator eingesetzt wird. Die grüne Phase wird so definiert, daß normalerweise alle Peak flow-Werte im Bereich zwischen 80 und 100\% des individuellen Bestwertes liegen und die Peak flow-Variabilität 20\% nicht überschreitet. Die Grenze zwischen Gelb und Rot wird mit 60\% angegeben. Obwohl die Patienten sich die Mühe machen sollen, den Peak flow auch nachmittags und nach Betastimulation zu kontrollieren, basiert die Interpretation faktisch nur auf dem ersten Peakflow am frühen Morgen, der üblicherweise der tiefste ist und damit die Indikation zum Phasenwechsel angibt.

Die 1997 erschienenen US-NAEPP [16] gehen nur von den Peak flow-Werten vor Betastimulation aus. Im Gegensatz zu den Southampton Guidelines aus dem Jahr 1989 [10] werden aber nicht nur die Peak flow-Werte des frühen Morgens, sondern im Falle einer signifikanten morgendlichen Obstruktion auch solche, die im Verlauf des Tages vor Betastimulation gewonnen werden, mitberücksichtigt. Dies hat den Vorteil, daß zumindest eine spontane Reversibilität in den Nachmittagsstunden erfaßt wird. Die US-NAEP sind in bezug auf das Peak flow-Monitoring jedoch widersprüchlich. „Asthma Action Pläne“, die den US-NAEP beiliegen, basieren auf den oben genannten Prinzipien. An anderer Stelle (Seite 108 der Originalpublikation) heißt es aber, daß im Falle einer signifikanten Obstruktion (Peak flow < $80 \%$ ) eine Betastimulation erfolgen sollte. Ein konsekutives Ansteigen des Peak flows auf $>80 \%$ des individuellen Bestwertes wird dann als leichte Exazerbation definiert und eine kurzfristige Verdoppelung der Dosis inhalativer Kortikosteroide empfohlen. Im Gegensatz zu den Aktionsplänen, die für die Patienten bestimmt sind, wird hier für die ärztliche Indikationsstellung zur medikamentösen Intervention plötzlich doch der Peak flow nach Betastimulation als entscheidend angeben.

Alle genannten Ampelschemata zur Selbsterkennung von Asthma-Exazerbationen durch Patienten sind dadurch gekennzeichnet, daß wesentliche Informationen verloren gehen, weil nicht die Peak flow-Werte vor und nach Betastimulation Eingang finden, sondern entweder nur die vor, bzw. nur die nach Applikation eines Betastimulators. Verschiedene Wege könnten aus dem aufgezeigten Dilemma führen:

1.) Unterteilt man die Gelbphase in zwei Unterbereiche (Gelb und Orange), kann die grüne Phase relativ hoch angesetzt werden. In den Los Angeles Guidelines [17] definieren Peak flow-Werte zwischen 90 und 100\% die grüne Phase. Es ist ausreichend, wenn der beste Peak flow-Wert des Tages vor Betastimulation in diesem Bereich zum Liegen kommt. Mit diesem Vorgehen ist gewährleistet, daß zumindest in der grünen Phase eine signifikante Obstruktion durch eine entzündliche Mukosaschwellung nicht übersehen wird. Bei Peak flow-Werten unter $90 \%$ läßt sich bei Anwendung dieses Schemas nicht erkennen, ob eine Reversibilität vorhanden ist, da naturgemäß zur Interpretation nur die Peak fow-Werte vor oder nach Bronchospasmolyse herangezogen werden können.
Dieser Ansatz wird u.a. auch in der Schweiz im Rahmen einer landesweiten Kampagne zur Asthma-Schulung propagiert. Die Angaben sind allerdings im Hinblick darauf unklar, ob ein Patient bereits auf einen einmaligen signifikanten Peak flowAbfall reagieren muß, oder erst dann, wenn tiefe Werte über ein bis zwei Tage persistieren. Eine ausreichende Evaluation des 4-Farben-Ampelschemas steht noch aus.

2.) Für viele Patienten wäre es zu kompliziert, wenn sie einer schriftlichen Empfehlung Folge leisten müßten, die auf der Interpretation der Peak flow-Werte vor und nach Betastimulation beruhte. Mit der Abgabe einer Graphik (Abb.1), die typische Peak flow-Verläufe veranschaulicht, läßt sich jedoch die Interpretation der Meßwerte deutlich vereinfachen [18]. Unterhalb der Grafik, in der zur jeweiligen Ampelphase zugehörigen Spalte, sind Zeichnungen der Bronchialquerschnitte und die dazugehörige Modifikation der medikamentösen Therapie vermerkt. Der Patient erkennt selbstständig, daß eine Obstruktion, die weitgehend reversibel ist, auf der Verkrampfung der Bronchialmuskeln beruht. Entsprechend werden dann kurzwirkende Betastimulatoren häufiger, oder alternativ langwirkende appliziert. Zusätzlich ist jedoch die Verdopplung der Dosis topischer Kortikosteroide zur Reduktion der zugrundeliegenden bronchialen Hyperreaktivität indiziert. Die Grafik zeigt weiterhin, daß im Falle fehlender bzw. ungenügender Reversibilität die Obstruktion auch auf einer Schwellung der Mukosa beruht, was meist den Einsatz oraler Kortikosteroide erfordert.

Trotz der das Verständnis erleichternden Grafik ist dies Peak flow-Interpretationshilfe sicher nicht für alle Patienten geeignet, die von einem Peak flow-Monitoring profitieren könnten. Obwohl in der täglichen Praxis bereits viele Patienten mit diesem Protokoll eigene Kompetenz erlangt haben, muß einschränkend festgehalten werden, daß auch hier bisher keine studienmäßige Evaluation erfolgt ist.

3.) Ein anderes Vorgehen wurde von Gibson [7] erfolgreich geprüft. 150 Patienten führten morgens und abends jeweils drei Peak flow-Messungen vor und nach Betastimulation durch. Aus den jeweils besten Werten wurde der mittlere Peak flow des individuellen Patienten errechnet. Mit hoher Sensitivität und nur geringer Zahl falsch Positiver zeigte ein einmaliger Abfall des Peak flows unter das 3s-Signifikanzniveau zuverlässig eine asthmatische Exazerbation an. Genauso zuverlässig war ein Abfall des Peak flows unter das 2sSignifikanzniveau, sofern diese Grenze bei 2 von 3 konsekutiven Messungen unterschritten wurde. Dieses Verfahren ist zumindest in der Initialphase aufgrund des damit verbundenen Rechnereinsatzes sehr aufwendig und damit wenig praxistauglich.

In Anbetracht der Tatsache, daß in den verschiedenen aktuellen Guidelines zum Teil divergierende Positionen bezüglich des Peak flow-Monitorings vertreten werden, wäre es wünschenswert, wenn zukünftige Guidelines auf einem gemeinsamen Konzept beruhten. Sie sollten in irgendeiner Form die Vorteile der Peak flow-Messungen vor bzw. nach Betastimulation vereinigen und einheitliche Grenzen für die Bereiche der Ampelfarben definieren. Darüber hinaus sind präzisere Angaben nötig, wie lange ein signifikanter Peak flow-Abfall andauern muß, bis therapeutische Konsequenzen gezogen werden. All diese Kriterien müßten in Studienprotokollen 
wissenschaftlich evaluiert werden. Diese Aufgabe dürfte allerdings sehr langwierig und zeitaufwendig sein.

Dieser Maximalforderung ist entgegenzuhalten, daß möglicherweise nicht alle Faktoren standardisiert werden können. $\mathrm{Zu}$ verschieden sind die Voraussetzungen des einzelnen Patienten u.a. im Hinblick auf die Eigenart seiner Erkrankung. Zukünftige Guidelines sollten deshalb angeben, in welchen Bereichen eine Individualisierung möglich ist, z.B. bei den Grenzen der Ampelfarben und der Intensität und Dauer der medikamentösen Intervention. Genauso wie nicht jeder Typ IDiabetiker für eine funktionelle Insulin-Therapie geeignet ist, muß auch bei Asthmatikern den intellektuellen Fähigkeiten Rechnung getragen werden. Eine dem entsprechende Individualisierung könnte so aussehen:

Patienten mit schwerem Asthma, entsprechendem Leidensdruck und ausreichenden kognitiven Fähigkeiten sollten regelmäßig den Peakflow kontrollieren und auf der Basis eines komplexeren Schemas (s.o.) interpretieren. Erweist sich dies als zu anspruchsvoll, wäre die alleinige Kontrolle des Peak flows am Nachmittag nach Betastimulation eine akzeptable Alternative. Gerade bei Patienten mit schwerem Asthma, vor allem wenn bereits eine Medikation mit langwirkenden Betastimulatoren etabliert worden ist, ist die Erkennung einer weder spontan, noch auf Betastimulation reversiblen Obstruktion von höchster Priorität. Dieser Ansatz wird durch eine aktuelle Arbeit aus der Gruppe von Ann Woolcock gestützt [19]. Sie ergab, daß asthmatische Exazerbationen am zuverlässigsten durch einen auf Betastimulatoren praktisch nicht reversiblen Peak flow-Abfall $\mathrm{zu}$ erkennen sind. Im Gegensatz dazu zeigten verminderte Peak flow-Werte in Episoden mit schlechter Asthma-Kontrolle (instabiles Asthma) eine deutliche Reversibilität. Beide Situationen, asthmatische Exazerbation und instabiles Asthma, äußern sich in Form vermehrter asthmatischer Beschwerden. Eine Exazerbation erfordert jedoch einen raschen Kortikosteroidstoß (anschließend kann mit der vorangehenden Therapiestufe fortgefahren werden), während im Falle eines instabilen Asthmas auf die nächst höhere Therapiestufe übergegangen werden muß. Bei einem schwergradigen Asthma, das immer mit einer gewissen Symptomatik verbunden ist, muß es als vordringlich angesehen werden, wenn Exazerbationen kurzfristig und sicher erkannt werden. In diesem Sinne erscheint mir der Ansatz, daß nur der Peak flow nach Betastimulation am Nachmittag gemessen und zur Interpretation mittels Ampelschema herangezogen wird, ein sinnvolles Minimum an Asthma-Monitoring bei Patienten mit schwerem Asthma bronchiale.

Bei einem Jugendlichen mit leicht- bis mittelgradigem Asthma dürfte hingegen der Peakflow am frühen Morgen vor Betastimulation von größerer Bedeutung sein. Wenn er absinkt, handelt es sich häufig um eine Zunahme der bronchialen Hyperreaktivität. In diesem Fall muß aber z.B. bei sportlichen Aktivitäten mit dem Auftreten eines Anstrengungsasthmas gerechnet werden. Für das Peak flow-Monitoring eines Jugendlichen mit leicht- bis mittelgradigem Asthma würde ich es deshalb als akzeptables Minimum ansehen, wenn lediglich die Werte am frühen Morgen vor Betastimulation registriert und zur Interpretation auf der Basis des Ampelschemas herangezogen werden.

\section{Literatur}

${ }^{1}$ GRASSIC. Effectiveness of routine self monitoring of peak flow in patients with asthma. BMJ 1994; 308: $564-567$

2 Ignacio-Garcia JM, Gonzales-Santos P. Asthma self-management education program by home monitoring of peak expiratory flow. Am J Respir Crit Care Med 1995; 151: 353 - 359

${ }^{3}$ Jain P, Kavuru MS, Emerman CL, Ahmad M. Utility of peak expiratory flow monitoring. Chest 1998; 114: $861-876$

${ }^{4}$ Magnussen H, Wettengel R. Das Peak-flow-Meter. Stellenwert in der pneumologischen Diagnostik. Med Klin 1993; 88: 720 - 723

${ }^{5}$ Bijl-Hofland ID, Cloosterman SGM, Folgering HTM, Akkermans $\mathrm{RP}$, Schayck van CP. Relation of the perception of airway obstruction to the severity of asthma. Thorax 1999; 54: 15 - 19

${ }^{6}$ Mclvor RA, Pizzichini E, Turner MO, Hussack P, Hargreave FE, Sears MR. Potential masking effects of salmeterol on airway inflammation in asthma. Am J Respir Crit Care Med 1998; 158: $924-930$

${ }^{7}$ Gibson PG, Wlodarczyk J, Hensley MJ, Murree-Allen K, Olson LG, Saltos N. Using quality-control analysis of peak expiratory flow recordings to guide therapy for asthma. Ann Intern Med 1995; 123: $488-492$

${ }^{8}$ Douma WR, Kerstjens HAM, Rooyackers JM, Koëter GH, Postma DS. Risk of overtreatment with current peak flow criteria in selfmanagement plans. Eur Respir J 1998; 12: 848 - 852

${ }^{9}$ Meijer RJ, Kerstjens HAM, Postma DS. Comparison of guidelines and selfmanagement plans in asthma. Eur Respir J 1997; 10: $1163-1172$

${ }^{10}$ Beasley R, Cushley M, Holgate ST. A self-management plan in the treatment of adult asthma. Thorax 1989; 44: 200 - 204

${ }^{11}$ Hargreave FE, Dolovich J, Newhouse MT. The assessment and treatment of asthma: A conference report. J Allergy Clin Immunol 1990; 85: 1098 - 1111

12 Reddel HK, Salome CM, Peat JK, Woolcock AJ. Which index of peak expiratory flow is most useful in the management of stable asthma. Am J Respir Crit Care Med 1995; 151: 1320 - 1325

${ }^{13}$ Jenkins CR, Woolcock AJ. Effect of prednisone and beclomethasone dipropionate on airway responsiveness in asthma. Thorax 1988; 43 : $378-382$

${ }^{14}$ International Consensus Report on diagnosis and management of asthma. Clin Exp Allergy, 1992. 22: 1 - 72

${ }^{15}$ Global Initiative for Asthma. Global strategy for asthma management and prevention. NHLBI/WHO workshop report. NIH Publication No. 95-3659,

${ }^{16}$ National Asthma Education and Prevention Program. Expert panel report 2. Guidelines for the diagnosis and management of asthma. NIH Publication No. 97-4051,

17 Mendoza GR. Peak flow monitoring. J Asthma 1991; 28: 161 177

${ }^{18}$ Rothe T. A comprehensive graphic approach to peak flow traffic light system interpretation. Eur Respir J 1997; 10: 961 - 962

${ }^{19}$ Reddel H, Ware S, Marks G, Salome C, Jenkins C, Woolcock A. Differences between asthma exacerbations and poor asthma control. Lancet 1999; 353: $364-369$

\section{Dr. Thomas Rothe}

Leitender Arzt

Luzerner Höhenklinik

3962 Montana-Vermala

Schweiz 